\title{
Radiofrequency Catheter Ablation Method or Stereotactic Body Radiotherapy in the Treatment of Ventricular Tachycardia
}

\author{
Yasemin Benderli Cihan \\ Department of Radiation Oncology, Kayseri City Education and Research Hospital, Turkey
}

Ventricular arrhythmias are a wide range of rhythm disturbances ranging from premature ventricularcomplex to ventricular fibrillation. It usually develops due to ischemic heart disease. It is one of the important causes of sudden cardiac death. ${ }^{1,2}$ Ventricular arrhythmias are assessed according to the symptoms, underlying pathological substrate, hemodynamic results, and long-term prognosis. Due to the complexity and gravity of these arrhythmias, timely treatment is important. ${ }^{1-3}$

Ventricular tachycardia (VT) prevalence is directly related to the study population, method of detection, and duration of observation. Treatment approaches for VT are determined according to the underlying heart disease and family history. The guidelines recommend medical treatment first in patients with VT, with implantable cardioverter defibrillator (ICD) implantation for secondary prevention. Although it is a lifesaving treatment, ICD shocks are painful and markedly reduce the patients' quality of life. Current guidelines recommend radiofrequency catheter ablation of VT in the patient group in which medical treatment is not effective, tolerated or not desired. In recent years, SBRT treatment method has also been tried. ${ }^{1-4}$ Catheter ablation procedure in ventricular arrhythmias was developed as inspired by the control of ventricular tachycardia by resection of myocardial scars in surgical studies. The most commonly used energy source in ablation was radiofrequency current. VT ablation was a difficult procedure and was associated with a significant risk of complications. ${ }^{4}$ In studies regarding the efficacy, safety, and long-term results of ablation therapy in over 20,000 patients who were ablated for VT, non-fatal peri-procedural major event was detected in $7.5 \%$ of the patients, vascular complication in $4.6 \%$, pericardial complication in $2.3 \%$, and stroke in $1 \%$. The 30 -day and 1-year mortality rates were $4.2 \%$ and $15.0 \%$, respectively.

Correspondence to: Dr. Yasemin Benderli Cihan, Department of Radiation Oncology, Kayseri City Education and Research Hospital, Turkey

E-mail: cihany@erciyes.edu.tr

Received: May 06, 2021; Revised: July 13, 2021;

Accepted: August 04, 2021

DOI: https://doi.org/10.29271/jcpsp.2021.09.1013
The 1-year incidence of re-ablation was found to be $10 \% .{ }^{4}$ In a meta-analysis in which VT ablation was compared with medical therapy in patients with ischemic heart disease, VT catheter ablation was reported to result in less VT recurrence than medical therapy, and catheter ablation was reported to be superior to standard medical therapy. ${ }^{3}$ Studies reported the longterm success rate to be between $41-49 \%{ }^{3,4}$

Although radiofrequency catheter ablation is the state-of-theart treatment available for drug-resistant ventricular arrhythmias in patients with structural heart disease and is increasingly being used, it is not curative for many patients. The reason is that both the applied dose and the volume treated are not sufficient. Studies have reported recurrence rate of up to $50 \%$ in six months. It has been reported that it has a poor prognosis associated with progressive heart failure and unavoidable VT. ${ }^{2}$ Therefore, new searches have begun in the treatment of VT. One of these is stereotactic body radiotherapy (SBRT).

In recent years, an electrophysiology-guided, non-invasive, cardiac SBRT has been used in the treatment of VT. It was first reported by Loo et al. in 2014 that it was successful in the treatment of VT. Later, studies on cardiac SBRT started to be concentrated. SBRT is the method that treats the tissue to be treated with high dose radiation and minimal damage to the normal adjacent tissue. Before applying SBRT, cardiac magnetic resonance imaging (MRI), computed tomography (CT), singlephoton emission CT (SPECT) or positron emission tomography (PET) are used to identify and localise abnormal myocardial damage causing $\mathrm{VT}^{5,6}{ }^{5,}$ The electrocardiographic imaging technique is combined with the imaging techniques taken previously. In this way, the hybrid/fused mapping method is made; and the area to be irradiated is determined. Fibrosis occurs in the irradiated area after 3-6 months due to radiation. In preclinical studies, the effective dose in SBRT is reported to be between 25 and $35 \mathrm{~Gy}$ in a single fraction. No increase in complications is reported when the dose is given between $35-40 .{ }^{6}$

On literature review, the preliminary results of ongoing studies and small case reports and series in SBRT in the short and medium term, seem promising. Considering the worldwide data, 50 patients have been treated so far. Loo et al. applied 25 Gy SBRT to a patient with VT. They reported that VT recurrence was observed in the ninth month in the patient who had no acute or chronic complications. ${ }^{5}$ Lloyd et al. have reported that 
there was a significant decrease in VT episodes in patients, who underwent SBRT ( $25 \mathrm{~Gy} /$ single fraction) due to refractory VT; but no change in left ventricular ejection fraction. There were mild inflammatory changes in the adjacent lung tissue in the third month of treatment. ${ }^{6}$ In a clinical study conducted by Chin et al., the apparent clinical benefit was 33\% in patients, who received SBRT (15-25 Gy) due to VT. It has been emphasised that there was no immediate effect as expected compared to other patient series. ${ }^{1}$ The multi-centre and multi-platform RAVENTA (NCT03867747) study is likely to give more clear information about the safety profile (side effect, quality of life, response to treatment, further treatment decision) of cardiac SBRT. Looking at the side effects, seen in the patients treated so far, mild or moderate pneumonia, pericardial effusion, heart failure, nausea, and valvular disease progression were detected. ${ }^{1,5,6}$

In conclusion, when antiarrhythmic drugs are ineffective, not tolerated, or not desired by the patient, catheter ablation is an important treatment option for patients with VA. Cardiac SBRT has also been tried in recent years. Although catheter ablation is the state-of-the-art treatment available for drug-resistant ventricular arrhythmias in patients with structural heart disease, it is not curative for many patients. Cardiac SBRT results are positive. However, studies investigating its efficacy and safety are limited in number. There is no study in the literature comparing both the treatment methods. Clinical studies are needed to compare which treatment method is better.

\section{REFERENCES}

1. Chin R, Hayase J, Hu P, Cao M, Deng J, Ajijola O, et al. 2020. Non-invasive stereotactic body radiation therapy for refractory ventriculararrhythmias: An institutional experience. J Interv Card Electrophysiol 2020; doi: 10.1007/s10840-020-00849-0.

2. Cuculich PS, Schill MR, Kashani R, Mutic S, Lang A, Cooper $D$, et al. Noninvasive cardiac radiation for ablation of ventricular tachycardia. N Engl J Med 2017; 377(24): 2325-36. doi: 10.1056/NEJMoa1613773

3. Patel D, Hasselblad V, Jackson KP, Pokorney SD, Daubert JP, Al-Khatib SM. Catheter ablation for ventricular tachycardia (VT) in patients with ischemic heart disease: A systematic review and a meta-analysis of randomised controlled trials. Journal of Interventional Cardiac Electrophysiology 2016; 45(2):111-7. doi: 10.1007/s10840-015-0083-4.

4. Yousuf OK, Zusterzeel R, Sanders W, Canos D, Dekmezian $C$, Silverman $\mathrm{H}$, et al. Trends and outcomes of catheter ablation for ventricular tachycardia in a community cohort. JACC Clin Electrophysiol 2018; 4(9):1189-99.

5. Loo BW, Soltys SG, Wang L, Lo A, Fahimian BP, lagaru A, et al. Stereotactic ablative radiotherapy for the treatment of refractory cardiac ventricular arrhythmia. Circ Arrhythm Electrophysiol 2015; 8(3):748-50. doi.org/10.1161/CIRCEP. 115.002765.

6. Lloyd MS, Wight J, Schneider F, Hoskins M, Attia T, Escott C, et al. Clinical experience of stereotactic body radiation for refractory ventriculartachycardia in advanced heart failure patients. Heart Rhythm 2020; 17(3):415-22. doi: 10.1016/ j.hrthm.2019.09.028. 